\title{
Lebesgues-Stieltjes Integrals of Fuzzy Stochastic Processes with Respect to Finite Variation Processes
}

\author{
Jinping Zhang, Lingli Luo, Xingmei Li, Xiaoying Wang \\ Department of Mathematics and Physics, North China Electric Power University, Beijing, China \\ Email: zhangjinping@ncepu.edu.cn, luolingli@ncepu.edu.cn, xingmeili@ncepu.edu.cn, \\ wangxiaoying@ncepu.edu.cn
}

Received 28 September 2015; accepted 27 November 2015; published 30 November 2015

Copyright (C) 2015 by authors and Scientific Research Publishing Inc.

This work is licensed under the Creative Commons Attribution International License (CC BY).

http://creativecommons.org/licenses/by/4.0/

(c) (1) Open Access

\section{Abstract}

Let $G=\left\{G_{t}(\omega), t \in[0, T]\right\}$ be a fuzzy stochastic process and $\left\{A_{t}(\omega), t \in[0, T]\right\}$ be a real valued finite variation process. We define the Lebesgue-Stieltjes integral denoted by $\int_{0}^{t} G_{s}(\omega) \mathrm{d} A_{s}(\omega)$ for each $t>0$ by using the selection method, which is direct, nature and different from the indirect definition appearing in some references. We shall show that this kind of integral is also measurable, continuous in time $t$ and bounded a.s. under the Hausdorff metric.

\section{Keywords}

Fuzzy Stochastic Process, Finite Variation Process, Fuzzy Stochastic Lebesgue-Stieltjes Integral, Measurability

\section{Introduction}

Recently, the theory of fuzzy functions has been developed quickly due to the measurements of various uncertainties arising not only from the randomness but also from the vagueness in some situations. For example, when considering wave height at time $t$ denoted by $f_{t}$, due to the influence of random factors and the limitations of the measurement tools and methods, we may not precisely know the height $f_{t}$. It is reasonable to consider the wave height as a fuzzy random variable on a probability space $(\Omega, \mathcal{F}, P)$.

Since Puri and Ralescu [1] (1986) defined fuzzy random variable, there had been many further topics such as expectations of fuzzy random variables, fuzzy stochastic processes, integrals of fuzzy stochastic processes, fuzzy stochastic differential equations etc. In order to study a fuzzy function $u$, it is natural and equivalent to 
study its $\alpha$-level set $[u]^{\alpha}$ for any $\alpha \in[0,1]$, where $[u]^{\alpha}$ is a set-valued function. Therefore, as usual, in order to explore the integrals of fuzzy stochastic processes, at first we can study the integrals of set-valued stochastic processes. Kisielewicz (1997) [2] used all selections to define the integral of a set-valued process as a nonempty closed subset of $L^{2}\left(\Omega, \mathcal{F}, P ; R^{n}\right)$, but did not consider its measurability. Based on Kisielewicz's work (1997) [2], Kim and Kim (1999) [3] studied some properties of this kind of integral. Jung and Kim (2003) [4] modified the definition in 1-dimensional Euclidean space $R$ so that the integral became a set-valued random variable. After the work [4], there are some references on set-valued integrals and fuzzy integrals. One may refer to papers such as [5]-[13] etc. and references therein. Zhang and Qi [14] (2013) considered the set-valued integral with respect to a finite variation process directly instead of taking the decomposable closure appearing in [4] [6] and other references. As a further work of [14], here we shall explore the integrals of fuzzy stochastic processes with respect to finite variation processes and prove the measurability and boundedness of this kind of integral, the continuity with respect to $t$ under the Hausdorff metric and its representation theorem.

This paper is organized as follows: in Section 2, we present some notions on set-valued random variables and fuzzy set-valued random variables; in Section 3, we shall give the definition of integral of fuzzy set-valued stochastic processes with respect to finite variation process and prove the measurability and $L^{2}$-boundedness which are necessary to our future work on fuzzy stochastic differential equations.

\section{Preliminaries}

We denote $N$ the set of all natural numbers, $R$ the set of all real numbers, $R^{d}$ the $d$-dimensional Euclidean space with the usual norm $\|\cdot\|, R^{+}$the set of all nonnegative numbers. Let $(\Omega, \mathcal{F}, P)$ be a complete probability space, $\left\{\mathcal{F}_{t}, t \in[0, T]\right\}$ a $\sigma$-field filtration satisfying the usual conditions such that $\mathcal{F}_{0}$ includes all $P$-null sets in $\mathcal{F}$. The filtration is non-decreasing and right continuous. Let $\mathcal{B}(E)$ be a Borel field of a topological space $E$.

Let $(\Omega, \mathcal{F}, P)$ be a complete probability space. $L^{p}\left(\Omega, \mathcal{F}, P ; R^{d}\right)$ (or brief $L^{p}\left(\Omega ; R^{d}\right)$ ) $(p \geq 1)$ the set of all $R^{d}$-valued Borel measurable functions $f: \Omega \rightarrow R^{d}$ such that the norm

$$
\begin{aligned}
& \|f\|_{p}=\left\{\int_{\Omega}\|f(\omega)\|^{p} \mathrm{~d} P\right\}^{\frac{1}{p}}, \text { if } 1 \leq p<\infty, \\
& \|f\|_{\infty}=\operatorname{esssup}_{\omega \in \Omega}\|f(\omega)\|, \text { if } p=\infty
\end{aligned}
$$

is finite. $f$ is called $L^{p}$-integrable if $f \in L^{p}\left(\Omega ; R^{d}\right)$.

Let $\mathcal{K}\left(R^{d}\right)$ (resp. $\mathcal{K}_{k}\left(R^{d}\right), \mathcal{K}_{k c}\left(R^{d}\right)$ ) be the family of all nonempty, closed (resp. nonempty compact, nonempty compact convex) subsets of $R^{d}$. For any $x \in R^{d}$ and $A \in \mathcal{K}\left(R^{d}\right)$, define the distance between $x$ and $A$ by $d(x, A)=\inf _{y \in A}\|x-y\|$. The Hausdorff metric $d_{H}$ on $\mathcal{K}\left(R^{d}\right)$ (cf. [15]) is defined by

$$
d_{H}(A, B) \doteq \max \left\{\sup _{a \in A} d(a, B), \sup _{b \in B} d(b, A)\right\} A, B \in \mathcal{K}\left(R^{d}\right) .
$$

Denote $\|A\|_{K} \doteq d_{H}(\{0\}, A)=\sup _{a \in A}\|a\|$.

For $A \subset R^{d}, x^{*} \in R^{d}$, the support function of $A$ is defined as follows:

$$
S\left(x^{*}, A\right)=\sup \left\{\left\langle x^{*}, x\right\rangle: x \in A\right\} .
$$

A set-valued function $F: \Omega \rightarrow \mathcal{K}\left(R^{d}\right)$ is said to be measurable if for any open set $O \subset R^{d}$, the inverse $F^{-1}(O):=\{\omega \in \Omega: F(\omega) \cap O \neq \varnothing\}$ belongs to $\mathcal{F}$. Such a function $F$ is called a set-valued random variable.

Let $\mathcal{M}\left(\Omega, \mathcal{F}, P ; \mathcal{K}\left(R^{d}\right)\right)$ (resp. $\left.\mathcal{M}\left(\Omega, \mathcal{F}, P ; \mathcal{K}_{c}\left(R^{d}\right)\right)\right), \mathcal{M}\left(\Omega, \mathcal{F}, P ; \mathcal{K}_{k c}\left(R^{d}\right)\right)$ ) be the family of all measurable $\mathcal{K}\left(R^{d}\right)$-valued (resp. $\mathcal{K}_{c}\left(R^{d}\right), \mathcal{K}_{k c}\left(R^{d}\right)$-valued) functions, briefly by $\mathcal{M}\left(\Omega, \mathcal{K}\left(R^{d}\right)\right.$ ) (resp. $\mathcal{M}\left(\Omega, \mathcal{F}, P ; \mathcal{K}_{c}\left(R^{d}\right)\right), \mathcal{M}\left(\Omega, \mathcal{F}, P ; \mathcal{K}_{k c}\left(R^{d}\right)\right)$. For $F \in \mathcal{M}\left(\Omega, K\left(R^{d}\right)\right)$, the family of all $L^{p}$-integrable selections is defined by

$$
S_{F}^{p}(\mathcal{F}):=\left\{f \in L^{p}\left(\Omega, \mathcal{F}, P ; R^{d}\right): f(\omega) \in F(\omega) \text { a.s. }\right\}, p \geq 1
$$


In the following, $S_{F}^{p}(\mathcal{F})$ is denoted briefly by $S_{F}^{p}$.

A set-valued random variable $F$ is said to be integrable if $S_{F}^{1}$ is nonempty. $F$ is called $L^{p}(p \geq 1)$ -integrably bounded if there exits $h \in L^{p}\left(\Omega, \mathcal{F}, P ; R^{d}\right)$ s.t. for all $x \in F(\omega),\|x\| \leq h(\omega)$ almost surely.

An $R^{d}$-valued stochastic process $f=\left\{f_{t}: t \geq 0\right\}$ (or denoted by $f=\{f(t): t \geq 0\}$ ) is defined as a function $f: R_{+} \times \Omega \rightarrow R^{d}$ with the $\mathcal{F}$-measurable section $f_{t}$, for $t \geq 0$. We say $f$ is measurable if $f$ is $\mathcal{B}\left(R_{+}\right) \otimes \mathcal{F}$-measurable. The process $f=\left\{f_{t}: t \geq 0\right\}$ is called $\mathcal{F}_{t}$-adapted if $f_{t}$ is $\mathcal{F}_{t}$-measurable for every $t \geq 0$. Let $\Sigma:=\bigcap_{t \geq 0}\left\{Z \in \mathcal{B}\left(R_{+}\right) \otimes \mathcal{F}: Z_{t} \in \mathcal{F}_{t}\right\}$, where $Z_{t}=\{\omega ;(t, \omega) \in Z\}$. We know that $\Sigma$ is a $\sigma$ -algebra on $R_{+} \times \Omega$. A function $f: R_{+} \times \Omega \rightarrow R^{d}$ is measurable and $\mathcal{F}_{t}$-adapted if and only if it is $\Sigma$ -measurable ([9]).

In a fashion similar to the $R^{d}$-valued stochastic processes, a set-valued stochastic process $F=\left\{F_{t}: t \geq 0\right\}$ is defined as a set-valued function $F: R_{+} \times \Omega \rightarrow \mathcal{K}\left(R^{d}\right)$ with $\mathcal{F}$-measurable section $F_{t}$ for $t \geq 0$. It is called measurable if it is $\mathcal{B}_{+} \otimes \mathcal{F}$-measurable, and $\mathcal{F}_{t}$-adapted if for any fixed $t, F_{t}(\cdot)$ is $\mathcal{F}_{t}$-measurable. $F=\left\{F_{t}: t \geq 0\right\}$ is measurable and $\mathcal{F}_{t}$-adapted if and only if it is $\Sigma$-measurable. $F=\left\{F_{t}: t \geq 0\right\}$ is called $L^{p}$-integrable if every $F_{t}$ is $L^{p}$-integrable.

Let $F\left(R^{d}\right)$ denote the family of all fuzzy sets $v: R^{d} \rightarrow[0,1]$ which satisfy the following two conditions (cf. [3] [6]):

1) The level set $v_{1}=\left\{x \in R^{d}: v(x)=1\right\} \neq \varnothing$;

2) Each $v$ is upper semi-continuous function, i.e. for each $\alpha \in(0,1]$, the level set $[v]^{\alpha} \doteq\left\{x \in R^{d}: v(x) \geq \alpha\right\}$ is a closed subset of $R^{d}$.

Let $F_{k}\left(R^{d}\right)$ denote the family of all fuzzy sets $v: R^{d} \rightarrow[0,1]$ which satisfy the above conditions 1$), 2$ ), and

3) The support set $[v]^{0} \doteq c l\left\{x \in R^{d}: v(x)>0\right\}$ is a compact set.

A fuzzy set $v$ is convex if

$$
v(\lambda x+(1-\lambda) y) \geq \min \{v(x), v(y)\} \text { for any } x, y \in R^{d}, \lambda \in[0,1] .
$$

It is know that $v$ is convex if and only if, for any $\alpha \in(0,1]$, the level set $[v]^{\alpha}$ is a convex subset of $R^{d}$. Let $F_{c}\left(R^{d}\right)$ denote the family of all convex fuzzy sets in $F\left(R^{d}\right)$, and $F_{k c}\left(R^{d}\right)$ be the subset of all convex fuzzy sets in $F_{k}\left(R^{d}\right)$. Define $d_{\infty}: F\left(R^{d}\right) \times F\left(R^{d}\right) \rightarrow[0, \infty)$ (cf. [1]) by the expression

$$
d_{\infty}(u, v):=\sup _{\alpha \in[0,1]} d_{H}\left([u]^{\alpha},[v]^{\alpha}\right) \text {. }
$$

We know that $d_{\infty}$ is a metric in $F\left(R^{d}\right)$ and $\left(F\left(R^{d}\right), d_{\infty}\right)$ a complete metric space (cf. [6] [3]). Moreover, for every $u, v, w, z \in F\left(R^{d}\right), \lambda \in R$, we have

$$
\begin{aligned}
& d_{\infty}(u+w, v+w)=d_{\infty}(u, v) ; \\
& d_{\infty}(u+v, w+z) \leq d_{\infty}(u, w)+d_{\infty}(v, z) ; \\
& d_{\infty}(\lambda u, \lambda v)=|\lambda| d_{\infty}(u, v) .
\end{aligned}
$$

Lemma 1. (cf. [16]) Let $B$ be a set and $\left\{B_{\alpha}, \alpha \in[0,1]\right\}$ be a family of subsets of $B$ such that

1) $B_{0}=B$;

2) $\alpha \leq \beta$ implies $B_{\beta} \subseteq B_{\alpha}$;

3) $\alpha_{1} \leq \alpha_{2} \leq \cdots, \lim _{n \rightarrow \infty} \alpha_{n}=\alpha$ implies $B_{\alpha}=\bigcap_{i=1}^{\infty} B_{\alpha_{i}}$.

Then the function $\phi: B \rightarrow[0,1]$ defined by $\phi(x)=\sup \left\{\alpha \in[0,1]: x \in B_{\alpha}\right\}$ has the property that $\{x \in B: \phi(x) \geq \alpha\}=B_{\alpha}$ for every $\alpha \in[0,1]$.

A mapping $G: \Omega \rightarrow F\left(R^{d}\right)$ is said to be measurable if $[G(\omega)]^{\alpha}: \Omega \rightarrow \mathcal{K}\left(R^{d}\right)$ is an set-valued random variable for each $\alpha \in(0,1]$. Such a mapping $G$ is called a fuzzy random variable (cf. [17]). Let $\mathcal{M}\left(\Omega, \mathcal{F}, P ; F\left(R^{d}\right)\right)$ (briefly by $\mathcal{M}\left(\Omega, F\left(R^{d}\right)\right)$ ) denote the family of all $\mathcal{F}$-measurable fuzzy random variables. As a similar manner, we have the notations $\mathcal{M}\left(\Omega, \mathcal{F}, P ; F_{k}\left(R^{d}\right)\right)$, and $\mathcal{M}\left(\Omega, \mathcal{F}, P ; F_{k c}\left(R^{d}\right)\right)$, or briefly by $\mathcal{M}\left(\Omega, F_{k}\left(R^{d}\right)\right)$ (resp. $\mathcal{M}\left(\Omega, F_{k c}\left(R^{d}\right)\right)$ ).

$G(t, \omega): \Omega \times[0, T] \rightarrow F\left(R^{d}\right)$ is called a fuzzy stochastic process if for any $t \in[0, T], G(t, \omega)$ is a fuzzy 
random variable. A fuzzy stochastic process $G(t, \omega)$ is said to be $\mathcal{F}_{t}$-adapted, if for every $\alpha \in[0,1]$, the set-valued function $[G(t, \omega)]^{\alpha}: \Omega \rightarrow \mathcal{K}\left(R^{d}\right)$ is $\mathcal{F}_{t}$-measurable for all $t \in[0, T]$. It is called measurable, if $[G(t, \omega)]^{\alpha}: \Omega \rightarrow \mathcal{K}\left(R^{d}\right)$ is a $\mathcal{B}([0, T]) \otimes \mathcal{F}$-measurable for all $\alpha \in[0,1]$.

A fuzzy stochastic process $G$ is called $L^{p}$-integrably bounded, if there exists a real-valued stochastic process $h \in L^{p}\left(\Omega \times[0, T], R^{d}\right)$, for any $\alpha \in[0,1]$ such that $\left\|[G(t, \omega)]^{\alpha}\right\|_{K} \leq h(t, \omega)$ for any $(t, \omega) \in \Omega \times[0, T]$. It is equivalent to that $\left\|[G]^{0}\right\|_{K} \in L^{p}\left(\Omega \times[0, T], R^{d}\right)$.

Let $L^{p}\left(\Omega, \mathcal{F}, P ; F\left(R^{d}\right)\right)$ denote the family of all measurable $F\left(R^{d}\right)$-valued $L^{p}$-integrably bounded fuzzy functions. Write for brevity by $L^{p}\left(\Omega, F\left(R^{d}\right)\right)$, where we consider $F, G \in L^{p}\left(\Omega, \mathcal{F}, P ; F\left(R^{d}\right)\right)$ as identical if $P\left([F]^{\alpha}=[G]^{\alpha}, \forall \alpha \in[0,1]\right)=1$. Let $L^{p}\left(\Omega \times[0, T], F\left(R^{d}\right)\right)$ denote the family of all $L^{p}$-integrably bounded $F\left(R^{d}\right)$-valued $\mathcal{F}_{t}$-adapted fuzzy stochastic processes.

Let $G: \Omega \rightarrow F\left(R^{d}\right)$ be a fuzzy random variable and $p \geq 1$, The following conditions are equivalent (cf. [15]):

1) $G \in L^{p}\left(\Omega, \mathcal{F}, P ; F\left(R^{d}\right)\right)$;

2) $[G]^{0} \in L^{p}\left(\Omega, \mathcal{F}, P ; \mathcal{K}\left(R^{d}\right)\right)$;

3) $\left\|[G]^{0}\right\|_{K} \in L^{p}\left(\Omega, \mathcal{F}, P ; R^{+}\right)$.

We define $\hat{\theta} \in F\left(R^{d}\right)$ as $\hat{\theta}=I_{\{0\}}$, where for $x \in R^{d}$, we have $I_{\{x\}}\{y\}=1$ if $x=y$ and $I_{\{x\}}\{y\}=0$ if $x \neq y$.

\section{Lebesgue-Stieltjes Integrals with Respect to Finite Variation Processes}

Let $(\Omega, \mathcal{F}, P)$ be a complete probability space equipped with the usual filtration $\left\{\mathcal{F}_{t}, t \in[0, T]\right\}$. Let $A=\left\{A_{t}, t \geq 0\right\}$ be a real valued $\mathcal{F}_{t}$-adapted measurable process with finite variation and continuous sample trajectories a.s. from the origin. That is to say, for each compact interval $[s, t] \subset[0, \infty)$ and any partition $\Delta=\left\{t_{1}, t_{2}, \cdots, t_{n}\right\}$ of $[s, t]$, the total variation

$$
V_{A}([s, t])=\sup _{\Delta} \sum_{i=1}^{n}\left|A_{t_{i}}(\omega)-A_{t_{i-1}}(\omega)\right|
$$

is finite and $A(0, \cdot)=0$ as. Then for any $T>0$, the process $A=\left\{A_{t}, t \geq 0\right\}$ can generate a random measure denoted by $\mu_{A}$ in the space $([0, T], \mathcal{B}([0, T]))$. For any $(s, t] \subset[0, T]$, let

$$
\mu_{A}((s, t]):=|A(t, \omega)|-|A(s, \omega)|
$$

where $A(t, \omega)=A^{+}(t, \omega)-A^{-}(t, \omega)$ is the decomposition of $A . A^{+}$and $A^{-}$are non-negative and non-decreasing processes. $|A(t, \omega)|=A^{+}(t, \omega)+A^{-}(t, \omega)$.

In the product space $(\Omega \times[0, T], \Sigma)$, Michta (2011) in [7] defined a measure as follows:

$$
v_{A}(C):=\int_{\Omega} \int_{[0, T]} I_{C}(t, \omega) \mu_{A}([0, T]) \mu_{A}(\mathrm{~d} t) P(\mathrm{~d} \omega)
$$

For $C \in \Sigma$, where $I_{C}$ is the index function. Then the set function $v$ is a finite measure in the measurable space $(\Omega \times[0, T], \Sigma)$ if and only if $\int_{\Omega}\left(\mu_{A}([0, T])\right)^{2} P(\mathrm{~d} \omega)<\infty$ (cf. [7]). In the following we always assume $\int_{\Omega}\left(\mu_{A}([0, T])\right)^{2} P(\mathrm{~d} \omega)<\infty$.

For $1 \leq p<\infty$, let $L^{p}\left(\Omega \times[0, T], \Sigma, v_{A} ; R^{d}\right)$ be the family of all $\Sigma$-measurable $R^{d}$-valued stochastic processes $f$ such that 


$$
\int_{\Omega \times[0, T]}\|f(\omega, t)\|^{p} \mathrm{~d} v_{A}<\infty .
$$

For any $f \in L^{p}\left(\Omega \times[0, T], \Sigma, v_{A} ; R^{d}\right)$ and $[s, t] \subset[0, T]$, the stochastic Lebesgue-Stieltjes integral $\int_{[s, t]} f(\tau) \mathrm{d} A_{\tau}$ is defined by the Bochner integral $\int_{[s, t]} f(\tau) \mu_{A}(\mathrm{~d} \tau)$ path-by-path. One can prove that the integral process $\left\{\int_{[0, t]} f(s) \mathrm{d} A_{s}, t \in[0, T]\right\}$ is $\Sigma$-measurable.

Lemma 2. (cf. [8]) Let $(E, \mathcal{B}, \mu)$ be a $\sigma$-finite measure space and $X$ a separable Banach space. If $\mathcal{B}$ is separable with respect to $\mu$, (i.e. there exists a countably generated sub-sigma algebra $\mathcal{B}_{0} \in \mathcal{B}$ such that for every $A \in \mathcal{B}$, there is $B \in \mathcal{B}_{0}$ satisfying $\left.\mu(A \triangle B)=0\right)$, then space $L^{p}(E ; X)(p \geq 1)$ is separable in norm.

From now on, we always assume the sigma-field $\mathcal{F}$ is separable with respect to $P$ such that the set-valued integral and fuzzy integral can be well defined.

Let $M^{p}\left(\Omega \times[0, T], \Sigma, v_{A} ; \mathcal{K}\left(R^{d}\right)\right)$ be the family of all $\Sigma$-measurable $\mathcal{K}\left(R^{d}\right)$-valued stochastic processes $F$ such that

$$
\int_{\Omega \times[0, T]}\|F(\omega, t)\|_{K}^{p} \mathrm{~d} v_{A}<\infty
$$

where $\|F(\omega, t)\|_{K}=\sup _{x \in F(\omega, t)}\|x\|$.

For any $F \in M^{p}\left(\Omega \times[0, T], \Sigma, v_{A} ; \mathcal{K}\left(R^{d}\right)\right)$, set

$$
S^{p}(F):=\left\{f \in M^{p}\left(\Omega \times[0, T], \Sigma, v_{A} ; R^{d}\right): f(\omega, t) \in F(\omega, t), v_{A}-a . e\right\} .
$$

Definition 1. (cf. [7]) For a set-valued stochastic process $F \in M^{p}\left(\Omega \times[0, T] ; \mathcal{K}_{k c}\left(R^{d}\right)\right)$ the set-valued stochastic Lebesgue-Stieltjes integral (over interval $[s, t]$ ) of $F$ with respect to the finite variation continuous process $A$ is the set

$$
\int_{[s, t]} F(\tau) \mathrm{d} A_{\tau}:=\left\{\int_{[s, t]} f(\tau) \mathrm{d} A_{\tau}: f \in S^{p}(F)\right\} .
$$

For some fuzzy stochastic process $G \in \mathcal{M}\left(\Omega \times[0, T], F\left(R^{d}\right)\right)$, it is natural to define the fuzzy integral of $G$ with respect to the finite variation process level-wise.

Let $M^{p}\left(\Omega \times[0, T], \Sigma, v_{A} ; F\left(R^{d}\right)\right)$ (or abbrev. as $M^{p}\left(\Omega \times[0, T], F\left(R^{d}\right)\right)$ ) be the family of all $\Sigma$-measurable $F\left(R^{d}\right)$-valued fuzzy stochastic processes $G$ such that

$$
\int_{\Omega \times[0, T]}\left\|[G(\omega, t)]^{0}\right\|_{K}^{p} \mathrm{~d} v_{A}<\infty,
$$

where $\left\|[G(\omega, t)]^{0}\right\|_{K}=\sup _{x \in[G(\omega, t)]^{0}\|x\|}$.

For a fuzzy stochastic process $G \in M^{1}\left(\Omega \times[0, T], F\left(R^{d}\right)\right)$, according to Lemma 1 and the properties of set-valued stochastic integrals, the Lebesgue-Stieltjes integral of $G$ (over interval $[s, t]$ ) can be defined level-wise.

Set

$$
\left[\int_{s}^{t} G(\tau, \omega) \mathrm{d} A_{\tau}(\omega)\right]^{\alpha}:=\int_{S}^{t}[G(\tau, \omega)]^{\alpha} \mathrm{d} A_{\tau}(\omega)=\left\{\int_{s}^{t} g_{\tau}(\omega) \mathrm{d} A_{\tau}(\omega), g \in S_{[G]^{\alpha}}^{1}\right\}
$$

for all $\alpha \in[0,1]$.

Definition 2. For a fuzzy stochastic process $G \in M^{1}\left(\Omega \times[0, T], F\left(R^{d}\right)\right)$ and any $0 \leq s<t \leq T$, the family $\left\{\left[\int_{s}^{t} G(\tau, \omega) \mathrm{d} A_{\tau}(\omega)\right]^{\alpha}, \alpha \in[0,1]\right\}$ defined by Equation (3) can determine an $F\left(R^{d}\right)$-valued function denoted by $\int_{0}^{t} G(s, \omega) \mathrm{d} A_{s}$, such a fuzzy function is called the Lebesgue-Stieltjes integral (over interval $[s, t]$ ) of $G$ with respect to finite variation process $A(t)$. 
Theorem 1. ([12]) For $F \in M^{1}\left(\Omega \times[0, T], \Sigma, \mathcal{K}_{k c}\left(R^{d}\right) ; v_{A}\right),[s, t] \subset[0, T]$ and $\omega \in \Omega$, the LebesgueStieltjes integral $\int_{s}^{t} F_{\tau}(\omega) \mathrm{d} A_{\tau}(\omega)$ is a compact and convex subset of $R^{d}$.

Lemma 3. (cf. [18]) Let $(\Omega, \mathcal{F}, P)$ be a probability space, $X$ a separable Banach space. For random variables $F_{1}, F_{2}$, both the support function $S\left(x^{*}, F(\omega)\right)\left(x^{*} \in X^{*}\right)$ and the metric $d_{H}\left(F_{1}(\omega), F_{2}(\omega)\right)$ are $\mathcal{F}$ -measurable.

Lemma 4. (cf. [14]) Let $A_{t}(\omega)$ be an R-valued stochastic process with finite variation. For $F \in L^{2}\left(\Omega \times[0, T], \Sigma, v_{A} ; \mathcal{K}_{k c}\left(R^{d}\right)\right)$ and $[s, t] \subset[0, T]$, we have

1) $\int_{s}^{t} \alpha F_{\tau} \mathrm{d} A_{\tau}=\alpha \int_{s}^{t} F_{\tau} \mathrm{d} A_{\tau}, \alpha \in[0,1]$;

2) $S\left(x^{*}, \int_{s}^{t} F_{\tau} \mathrm{d} A_{\tau}\right)=\int_{s}^{t} S\left(x^{*}, F_{\tau}\right) \mathrm{d} A_{\tau}, x^{*} \in R^{d}$.

Lemma 5. (cf. [18]) Let $(\Omega, \mathcal{F})$ be a measurable space, $X$ a separable Banach space. Taking $F: \Omega \rightarrow \mathcal{K}(X)$ and for any $x^{*} \in X^{*}$, assume $S\left(x^{*}, F\right)$ is measurable. Then if one of the following conditions is satisfied:

1) $X^{*}$ is separable;

2) for any $\omega \in \Omega, F(\Omega) \in \mathcal{K}_{k c}(X)$.

We obtain that $F$ is a set-valued random variable.

From Lemma 3 and Lemma 5, when $X=R^{d}$, taking $F(\omega) \in \mathcal{K}_{k c}\left(R^{d}\right)$, then for any $x^{*} \in R^{d}, F(\cdot)$ is measurable if and only if $S\left(x^{*}, F(\cdot)\right)$ is $\mathcal{F}_{t}$-measurable.

Lemma 6. (cf. [19]) Let $(\Omega, \mathcal{F})$ be a measurable space, $X$ a separable metrizable space, and $Y$ a metrizable space. Then every Caratheodory function $f: \Omega \times X \rightarrow Y$ (i.e. for each $x \in X$, the function $f(\cdot, x): \Omega \rightarrow Y$ is $\mathcal{F}$-measurable and for each $\omega \in \Omega$, the function $f(\omega, \cdot): X \rightarrow Y$ is continuous) is $(\mathcal{F} \otimes \mathcal{B}(X))$-measurable.

Theorem 2. Let $G \in M^{1}\left(\Omega \times[0, T], \Sigma, v_{A} ; F_{k c}\left(R^{d}\right)\right)$. Then for each $t \in[0, T]$, the fuzzy stochastic integral $\int_{0}^{t} G(s, \omega) \mathrm{d} A_{s}(\omega)$ is $\mathcal{F}_{t}$-measurable. Furthermore, the mapping $\psi(t, \omega)=\int_{0}^{t} G(s, \omega) \mathrm{d} A_{s}(\omega)$ is $\mathcal{B}([0, T]) \otimes \mathcal{F}$-measurable.

Proof. Taking $G \in M^{1}\left(\Omega \times[0, T], \Sigma, v_{A} ; F_{k c}\left(R^{d}\right)\right)$, then for each $\alpha \in[0,1]$, the mapping $[G(t, \omega)]^{\alpha}: \Omega \times[0, T] \rightarrow \mathcal{K}\left(R^{d}\right)$ is $\Sigma$-measurable. For any $x^{*} \in R^{d}$, by Lemma 3, the support function $S\left(x^{*},[G(t, \omega)]^{\alpha}\right)$ is $\Sigma$-measurable too. By Lemma 4, we have $S\left(x^{*}, \int_{0}^{t}[G(s, \omega)]^{\alpha} \mathrm{d} A_{s}\right)=\int_{0}^{t} S\left(x^{*},[G(s, \omega)]^{\alpha}\right) \mathrm{d} A_{s}$. Since the real-valued Lebesgue-Stieltjes integral $\int_{0}^{t} S\left(x^{*},[G(s, \omega)]^{\alpha}\right) \mathrm{d} A_{s}$ is a Carathedory function, then by Lemma 6, we obtain that $S\left(x^{*}, \int_{0}^{t}[G(s, \omega)]^{\alpha} \mathrm{d} A_{s}\right)$ is $\Sigma$-measurable. Therefore, by Lemma 5, for each $\alpha \in[0,1]$, the mapping $\int_{0}^{t}[G(s, \omega)]^{\alpha} \mathrm{d} A_{s}(\omega): \Omega \times[0, T] \rightarrow \mathcal{K}_{k c}\left(R^{d}\right)$ is $\mathcal{B}([0, T]) \otimes \mathcal{F}$-measurable and $\mathcal{F}_{t}$-adapted, which means the integral $\psi(t, \omega)=\int_{0}^{t} G(s, \omega) \mathrm{d} A_{s}(\omega)$ is $\mathcal{B}([0, T]) \otimes \mathcal{F}$-measurable and $\mathcal{F}_{t}$-adapted.

Theorem 3. Let $G \in M^{2}\left(\Omega \times[0, T], F_{k c}\left(R^{d}\right)\right)$. Then for any $t \in[0, T], \int_{0}^{t} G(s, \omega) \mathrm{d} A_{s}(\omega) \in L^{2}\left(\Omega, F\left(R^{d}\right)\right)$.

Proof. By Theorem 2, for any $t \in[0, T], \int_{0}^{t} G(s, \omega) \mathrm{d} A_{s}(\omega)$ is $\mathcal{F}_{t}$-measurable. We will show that for any $\omega \in \Omega, \quad t \in[0, T], \quad E\left[\left\|\int_{0}^{t}[G(s, \omega)]^{0} \mathrm{~d} A_{s}(\omega)\right\|_{K}^{2}\right]<\infty$.

For any $g \in S_{[G]^{0}}^{2}$, 


$$
\begin{aligned}
\left\|\int_{0}^{t} g(s, \omega) \mathrm{d} A_{s}(\omega)\right\|^{2} & \leq \mu_{A}([0, T]) \int_{0}^{t}\left\|g_{s}(\omega)\right\|^{2} \mathrm{~d} A_{s}(\omega) \\
& \leq \mu_{A}([0, T]) \int_{0}^{t}\left\|[G(s, \omega)]^{0}\right\|_{K}^{2} \mathrm{~d} A_{s}(\omega)
\end{aligned} .
$$

Then

$$
\sup _{g \in S_{[G]^{0}}}\left\|\int_{0}^{t} g_{s}(\omega) \mathrm{d} A_{s}(\omega)\right\|^{2} \leq \mu_{A}([0, T]) \int_{0}^{t}\left\|[G(s, \omega)]^{0}\right\|_{K}^{2} \mathrm{~d} A_{s} .
$$

Hence,

$$
\begin{aligned}
E\left[\left\|\int_{0}^{t}[G(s, \omega)]^{0} \mathrm{~d} A_{s}\right\|_{K}^{2}\right] & \leq E\left[\mu_{A}([0, T]) \int_{0}^{t}\left\|[G(s, \omega)]^{0}\right\|_{K}^{2} \mathrm{~d} A_{s}\right] \\
& \leq \int_{\Omega \times[0, T]}\left\|[G(s, \omega)]^{0}\right\|_{K}^{2} \mathrm{~d} v_{A}<\infty,
\end{aligned}
$$

which means $\int_{0}^{t} G(s, \omega) \mathrm{d} A_{s}(\omega) \in L^{2}\left(\Omega, F\left(R^{d}\right)\right)$.

Theorem 4. Let $G \in M^{1}\left(\Omega \times[0, T], F_{k c}\left(R^{d}\right)\right)$. Then for any $\omega \in \Omega, \int_{0}^{t} G(s, \omega) \mathrm{d} A_{s}(\omega)$ is continuous with respect to $t$ under the metric $d_{\infty}$.

Proof. Let $0 \leq r<t \leq T$, for any $\omega \in \Omega$, we have

$$
\int_{0}^{t} G(s, \omega) \mathrm{d} A_{s}(\omega)=\int_{0}^{r} G(s, \omega) \mathrm{d} A_{s}(\omega)+\int_{r}^{t} G(s, \omega) \mathrm{d} A_{s}(\omega) .
$$

Then

$$
\begin{aligned}
& d_{\infty}\left(\int_{0}^{r} G(s, \omega) \mathrm{d} A_{s}(\omega), \int_{0}^{t} G(s, \omega) \mathrm{d} A_{s}(\omega)\right) \\
& =d_{\infty}\left(\int_{0}^{r} G(s, \omega) \mathrm{d} A_{s}(\omega), \int_{0}^{r} G(s, \omega) \mathrm{d} A_{s}(\omega)+\int_{r}^{t} G(s, \omega) \mathrm{d} A_{s}(\omega)\right) \\
& \leq d_{\infty}\left(\int_{0}^{r} G(s, \omega) \mathrm{d} A_{s}(\omega), \int_{0}^{r} G(s, \omega) \mathrm{d} A_{s}(\omega)\right)+d_{\infty}\left(\hat{\theta}, \int_{r}^{t} G(s, \omega) \mathrm{d} A_{s}(\omega)\right) \\
& =d_{\infty}\left(\hat{\theta}, \int_{r}^{t} G(s, \omega) \mathrm{d} A_{s}(\omega)\right)=\sup _{\alpha \in[0,1]} d_{H}\left(\{0\}, \int_{r}^{t}[G(s, \omega)]^{\alpha} \mathrm{d} A_{s}(\omega)\right) \\
& \leq \sup _{\alpha \in[0,1]} \int_{r}^{t} d_{H}\left(\{0\},[G(s, \omega)]^{\alpha}\right) \mathrm{d} A_{s}(\omega) \leq \int_{r}^{t} \sup _{\alpha \in[0,1]} d_{H}\left(\{0\},[G(s, \omega)]^{\alpha}\right) \mathrm{d} A_{s}(\omega) \\
& \leq \int_{r}^{t} \sup _{\alpha \in[0,1]}\left\|[G(s, \omega)]^{\alpha}\right\|_{K} \mathrm{~d} A_{s}(\omega)=\int_{r}^{t}\left\|[G(s, \omega)]^{0}\right\|_{K} \mathrm{~d} A_{s}(\omega)<\infty .
\end{aligned}
$$

For any $\omega \in \Omega$, we have

$$
\lim _{r \rightarrow t} \int_{r}^{t}\left\|[G(s, \omega)]^{0}\right\|_{K} \mathrm{~d} A_{s}(\omega)=0 .
$$

Then for all $\omega \in \Omega, \int_{0}^{t} G(s, \omega) \mathrm{d} A_{s}(\omega)$ is left continuous for $t \in[0, T]$ under the metric $d_{\infty}$. Similarly, we can prove that $\int_{0}^{t} G(s, \omega) \mathrm{d} A_{s}(\omega)$ is a right continuous for $t \in[0, T]$. Therefore it is continuous in $t$ with respect to $d_{\infty}$.

Lemma 7. Let fuzzy stochastic process $G \in L^{1}\left(\Omega \times[0, T], F_{k c}\left(R^{d}\right)\right)$. Then for each $\alpha \in[0,1]$, there exists a sequence $\left\{f_{\alpha}^{i}: i \in N\right\} \subset S_{[G]^{\alpha}}^{1}$, such that for every $t \in[0, T]$,

$$
S_{[G]^{\alpha}}^{1}=c l\left\{f_{\alpha}^{i}: i \in N\right\},
$$

where the closure is taken in $L^{1}\left(\Omega \times[0, T], R^{d} ; v_{A}\right)$.

Proof. Since $\mathcal{F}$ is separable with respect to probability measurable $P$, we have that $\mathcal{B}([0, T]) \otimes \mathcal{F}$ is separable with respect to product measure $\lambda \times P$. By Lemma 2, $L^{1}\left(\Omega \times[0, T], R^{d} ; \lambda \times P\right)$ is separable. It can be obtained that $L^{1}\left(\Omega \times[0, T], \Sigma, R^{d} ; v_{A}\right)$ is separable under the norm $v_{A}$. So that for any $\alpha \in[0,1], S_{[G]^{\alpha}}^{1}$ is 
separable since it is a closed subset of $L^{1}\left(\Omega \times[0, T], \Sigma, R^{d} ; v_{A}\right)$. Then there exists a sequence $\left\{f_{\alpha}^{i}: i \in N\right\} \subset S_{[G]^{\alpha}}^{1}$,

$$
S_{[G]^{\alpha}}^{1}=\operatorname{cl}\left\{f_{\alpha}^{i}: i \in N\right\}
$$

Theorem 5. For a fuzzy set-valued stochastic process $G \in M^{1}\left(\Omega \times[0, T], F_{k c}\left(R^{d}\right)\right)$ and any $\alpha \in[0,1]$, there exists a sequence $\left\{f_{\alpha}^{i}: i \in N\right\} \subset S_{[G]^{\alpha}}^{1}$ such that

$$
[G(t, \omega)]^{\alpha}=c l\left\{f_{\alpha}^{i}(t, \omega): i \in N\right\} \text { a.e. }(t, \omega)
$$

and for each $t$

$$
\int_{0}^{t}[G(s, \omega)]^{\alpha} \mathrm{d} A_{s}(\omega)=c l\left\{\int_{0}^{t} f_{\alpha}^{i}(s, \omega) \mathrm{d} A_{s}(\omega): i \in N\right\} \text { a.s. }
$$

where " $c l$ " denotes the closure in $R^{d}$.

Proof. For each $\alpha \in[0,1]$, by Lemma 7, there exists a sequence $\left\{f_{\alpha}^{n}: n \in N\right\} \subset L^{1}\left(\Omega \times[0, T], \Sigma, R^{d} ; v_{A}\right)$ such that

$$
S_{[G]^{\alpha}}^{1}=c l\left\{f_{\alpha}^{i}: i \in N\right\},
$$

where the closure is taken in $L^{1}\left(\Omega \times[0, T], \Sigma, R^{d} ; v_{A}\right)$.

For each $\alpha \in[0,1]$, by Castaing represent theorem (cf. [15] [20]), there exists a sequence $\left\{g_{\alpha}^{j}: j \in N\right\} \subset S_{[G]^{\alpha}}^{1}$ such that

$$
[G(t, \omega)]^{\alpha}=c l\left\{g_{\alpha}^{j}(t, \omega): j \in N\right\} \text { for all }(t, \omega) \in[0, T] \times \Omega .
$$

At first we will show that

$$
[G(t, \omega)]^{\alpha} \subset c l\left\{f_{\alpha}^{i}(t, \omega): i \in N\right\} \text { a.e. }(t, \omega) .
$$

In fact, taking $g_{\alpha}^{j} \in S_{[G]^{\alpha}}^{1}$, there exists a sequence $\left\{f_{\alpha}^{i_{k}}\right\}$ such that

$$
\left\|f_{\alpha}^{i_{K}}-g_{\alpha}^{j}\right\|_{L^{1}\left(\Omega \times[0, T], \Sigma, \nu_{A}, R^{d}\right)} \rightarrow 0,
$$

then there exists a subsequence $\left\{f_{\alpha}^{i_{k_{j}}}\right\}$ such that

$$
\left\|f_{\alpha}^{i_{k_{j}}}(t, \omega)-g_{\alpha}^{j}(t, \omega)\right\|_{R^{d}} \rightarrow 0 \text { a.e }(t, \omega) .
$$

Therefore

$$
[G(t, \omega)]^{\alpha} \subset \operatorname{cl}\left\{f_{\alpha}^{i}(t, \omega): i \in N\right\} \text { a.e }(t, \omega) .
$$

On the other hand

$$
\operatorname{cl}\left\{f_{\alpha}^{i}(t, \omega): i \in N\right\} \subset[G(t, \omega)]^{\alpha} \text { a.e. }(t, \omega),
$$

since $[G(t, \omega)]^{\alpha}$ is closed and $f_{\alpha}^{i} \in S_{[G]^{\alpha}}^{1}$, which yields

$$
[G(t, \omega)]^{\alpha}=\operatorname{cl}\left\{f_{\alpha}^{i}(t, \omega): i \in N\right\} \text { a.e }(t, \omega) .
$$

Since

$$
\int_{0}^{t}[G(s, \omega)]^{\alpha} \mathrm{d} A_{s}(\omega)=\left\{\int_{0}^{t} g_{\alpha}(s, \omega) \mathrm{d} A_{s}(\omega): g_{\alpha} \in S_{[G]^{\alpha}}^{1}\right\}
$$

is closed and $f_{\alpha}^{i} \in S_{[G]^{\alpha}}^{1}$, then for each $t$

$$
\operatorname{cl}\left\{\int_{0}^{t} f_{\alpha}^{i}(s, \omega) \mathrm{d} A_{s}(\omega): i \in N\right\} \subset \int_{0}^{t}[G(s, \omega)]^{\alpha} \mathrm{d} A_{s}(\omega) \text { a.s. }
$$


For any $g_{\alpha} \in S_{[G]^{\alpha}}^{1}$, there exists a sequence $\left\{f_{\alpha}^{i_{k}}: k \in N\right\} \subset\left\{f^{i}: i \in N\right\}$ such that

$$
\left\|f_{\alpha}^{i_{k}}-g_{\alpha}\right\|_{L^{1}\left(\Omega \times[0, T], \Sigma, v_{A}, R^{d}\right)} \rightarrow 0 .
$$

Then for each $t$,

$$
\int_{0}^{t} g_{\alpha}(s, \omega) \mathrm{d} A_{s} \in \operatorname{cl}\left\{\int_{0}^{t} f_{\alpha}^{i}(s, \omega) \mathrm{d} A_{s}: i \in N\right\} \text { a.s. }
$$

which means

$$
\int_{0}^{t}[G(s, \omega)]^{\alpha} \mathrm{d} A_{s}(\omega) \subset c l\left\{\int_{0}^{t} f_{\alpha}^{i}(s, \omega) \mathrm{d} A_{s}(\omega): i \in N\right\} \text { a.s. }
$$

(7) together with (8) yields

$$
\operatorname{cl}\left\{\int_{0}^{t} f_{\alpha}^{i}(s, \omega) \mathrm{d} A_{s}(\omega): i \in N\right\}=\int_{0}^{t}[G(s, \omega)]^{\alpha} \mathrm{d} A_{s}(\omega) \text { a.s. }
$$

Lemma 8. (cf. [15]) Let $F \in \mathcal{M}\left(\Omega, \mathcal{K}\left(R^{d}\right)\right), \phi: \Omega \times X \rightarrow \bar{R}=[-\infty,+\infty]$ satisfy: for fixed $\omega \in \Omega, \quad \phi(\omega, \cdot)$ is continuous with respect to $x$, for fixed $x \in X, \phi(\omega, x)$ is measurable with respect to $\omega$, then there exists an $f_{0} \in S_{F}^{p}$ such that $\int_{\Omega} \phi(\omega, f(\omega)) \mathrm{d} \mu<\infty$, then we have

$$
\inf _{f \in S_{F}^{p}} \int_{\Omega} \phi(\omega, f(\omega)) \mathrm{d} \mu=\int_{\Omega} \inf _{f \in F(\omega)} \phi(\omega, f(\omega)) \mathrm{d} \mu .
$$

Theorem 6. Let $F, G \in M^{1}\left(\Omega \times[0, T], F_{k c}\left(R^{d}\right)\right)$. Then for any $t \in[0, T]$,

$$
d_{\infty}\left(\int_{0}^{t} F(s, \omega) \mathrm{d} A_{s}(\omega), \int_{0}^{t} G(s, \omega) \mathrm{d} A_{s}(\omega)\right) \leq \int_{0}^{t} d_{\infty}(F(s, \omega), G(s, \omega)) \mathrm{d} A_{s}(\omega) \text { a.s. }
$$

Proof. Let $\phi_{t}(\omega)=\int_{0}^{t} F_{s}(\omega) \mathrm{d} A_{s}(\omega), \psi_{t}(\omega)=\int_{0}^{t} G_{s}(\omega) \mathrm{d} A_{s}(\omega)$. By Theorem 5, we can obtain that for each $\alpha \in[0,1]$, there exist sequences $\left\{f_{\alpha}^{i}: i \geq 1\right\} \subseteq S_{[F]^{\alpha}}^{1}$ and $\left\{g_{\alpha}^{j}: j \geq 1\right\} \subseteq S_{[G]^{\alpha}}^{1}$ such that $[F(t, \omega)]^{\alpha}=c l\left\{f_{\alpha}^{i}(t, \omega): i \geq 1\right\}$ a.e. $(t, \omega),[G(s, \omega)]^{\alpha}=c l\left\{g_{\alpha}^{j}(t, \omega): j \geq 1\right\}$ a.e. $(t, \omega)$. For each $t$,

$$
\int_{0}^{t}[F(s, \omega)]^{\alpha} \mathrm{d} A_{s}(\omega)=c l\left\{\int_{0}^{t} f_{\alpha}^{i}(s, \omega) \mathrm{d} A_{s}(\omega): i \in N\right\} \text { a.s. }
$$

and

$$
\int_{0}^{t}[G(s, \omega)]^{\alpha} \mathrm{d} A_{s}(\omega)=c l\left\{\int_{0}^{t} g_{\alpha}^{j}(s, \omega) \mathrm{d} A_{s}(\omega): j \in N\right\} \text { a.s. }
$$

Therefore

$$
\begin{aligned}
& \inf _{y \in \int_{[}^{t}[G(s, \omega)]^{\alpha} \mathrm{d} A_{s}(\omega)}\left\|\int_{0}^{t} f_{\alpha}^{i}(s, \omega) \mathrm{d} A_{s}(\omega)-y\right\| \\
& =\inf _{j \geq 1}\left\|\int_{0}^{t} f_{\alpha}^{i}(s, \omega) \mathrm{d} A_{s}(\omega)-\int_{0}^{t} g_{\alpha}^{j}(s, \omega) \mathrm{d} A_{s}(\omega)\right\| \\
& \leq \inf _{j \geq 1} \int_{0}^{t}\left\|f_{\alpha}^{i}(s, \omega)-g_{\alpha}^{j}(s, \omega)\right\| \mathrm{d} A_{s}(\omega) \text { a.s. }
\end{aligned}
$$

By Lemma 8, we have

$$
\inf _{j \geq 1} \int_{0}^{t}\left\|f_{\alpha}^{i}(s, \omega)-g_{\alpha}^{j}(s, \omega)\right\| \mathrm{d} A_{s}(\omega)=\int_{0}^{t} \inf _{j \geq 1}\left\|f_{\alpha}^{i}(s, \omega)-g_{\alpha}^{j}(s, \omega)\right\| \mathrm{d} A_{s}(\omega) a . s .
$$

Then 


$$
\begin{aligned}
& \int_{0}^{t} \inf _{j \geq 1}\left\|f_{\alpha}^{i}(s, \omega)-g_{\alpha}^{j}(s, \omega)\right\| \mathrm{d} A_{s}(\omega) \\
& \leq \int_{0}^{t} \operatorname{supinf}_{i \geq 1}\left\|f_{\alpha}^{i}(s, \omega)-g_{\alpha}^{j}(s, \omega)\right\| \mathrm{d} A_{s}(\omega) \text { a.s. } \\
& \leq \int_{0}^{t} d_{H}\left([F(s, \omega)]^{\alpha},[G(s, \omega)]^{\alpha}\right) \mathrm{d} A_{s}(\omega) \text { a.s. } \\
& \leq \int_{0}^{t} \sup _{\alpha \in[0,1]} d_{H}\left([F(s, \omega)]^{\alpha},[G(s, \omega)]^{\alpha}\right) \mathrm{d} A_{s}(\omega) \text { a.s. } \\
& =\int_{0}^{t} d_{\infty}(F(s, \omega), G(s, \omega)) \mathrm{d} A_{s}(\omega) .
\end{aligned}
$$

Then

$$
\operatorname{supinf}_{j \geq 1}\left\|\int_{0}^{t} f_{\alpha}^{i}(s, \omega) \mathrm{d} A_{s}(\omega)-\int_{0}^{t} g_{\alpha}^{j}(s, \omega) \mathrm{d} A_{s}(\omega)\right\| \leq \int_{0}^{t} d_{\infty}(F(s, \omega), G(s, \omega)) \mathrm{d} A_{s}(\omega) \text { a.s. }
$$

Similarly, we have

$$
\operatorname{supinf}_{j \geq 1}\left\|\int_{i \geq 1}^{t} g_{\alpha}^{j}(s, \omega) \mathrm{d} A_{s}(\omega)-\int_{0}^{t} f_{\alpha}^{i}(s, \omega) \mathrm{d} A_{s}(\omega)\right\| \leq \int_{0}^{t} d_{\infty}(F(s, \omega), G(s, \omega)) \mathrm{d} A_{s}(\omega) \text { a.s. }
$$

Then for each $\alpha \in[0,1]$,

$$
d_{H}\left(\int_{0}^{t}[F(s, \omega)]^{\alpha} \mathrm{d} A_{s}(\omega), \int_{0}^{t}[G(s, \omega)]^{\alpha} \mathrm{d} A_{s}(\omega)\right) \leq \int_{0}^{t} d_{\infty}(F(s, \omega), G(s, \omega)) \mathrm{d} A_{s}(\omega) \text { a.s. }
$$

Therefore

$$
\sup _{\alpha \in[0,1]} d_{H}\left(\int_{0}^{t}[F(s, \omega)]^{\alpha} \mathrm{d} A_{s}(\omega), \int_{0}^{t}[G(s, \omega)]^{\alpha} \mathrm{d} A_{s}(\omega)\right) \leq \int_{0}^{t} d_{\infty}(F(s, \omega), G(s, \omega)) \mathrm{d} A_{s}(\omega) \text { a.s. }
$$

Hence

$$
d_{\infty}\left(\int_{0}^{t} F(s, \omega) \mathrm{d} A_{s}(\omega), \int_{0}^{t} G(s, \omega) \mathrm{d} A_{s}(\omega)\right) \leq \int_{0}^{t} d_{\infty}(F(s, \omega), G(s, \omega)) \mathrm{d} A_{s}(\omega) a . s .
$$

Theorem 7. Let $F, G \in M^{2}\left(\Omega \times[0, T], F_{k c}\left(R^{d}\right)\right)$. Then for each $t \in[0, T]$, we have

$$
d_{\infty}^{2}\left(\int_{0}^{t} F(s, \omega) \mathrm{d} A_{s}(\omega), \int_{0}^{t} G(s, \omega) \mathrm{d} A_{s}(\omega)\right) \leq \mu_{A}([0, T]) \int_{0}^{t} d_{\infty}^{2}(F(s, \omega), G(s, \omega)) \mathrm{d} A_{s}(\omega) \text { a.s. }
$$

Proof. For any $\alpha \in[0,1]$, we have

$$
\begin{aligned}
& \inf _{j \geq 1}\left\|\int_{0}^{t} f_{\alpha}^{i}(s, \omega) \mathrm{d} A_{s}(\omega)-\int_{0}^{t} g_{\alpha}^{j}(s, \omega) \mathrm{d} A_{s}(\omega)\right\|^{2} \\
& \leq \inf _{j \geq 1}\left\{\int_{0}^{t}\left\|f_{\alpha}^{i}(s, \omega)-g_{\alpha}^{j}(s, \omega)\right\| \mathrm{d} A_{s}(\omega)\right\}^{2} \\
& \leq \inf _{j \geq 1}\left\{\mu_{A}([0, T]) \int_{0}^{t}\left\|f_{\alpha}^{i}(s, \omega)-g_{\alpha}^{j}(s, \omega)\right\|^{2} \mathrm{~d} A_{s}(\omega)\right\} \text { a.s. }
\end{aligned}
$$

by Lemma 8, we have

$$
\begin{aligned}
& \inf _{j \geq 1}\left\{\mu_{A}([0, T]) \int_{0}^{t}\left\|f_{\alpha}^{i}(s, \omega)-g_{\alpha}^{j}(s, \omega)\right\|^{2} \mathrm{~d} A_{s}(\omega)\right\} \\
& =\mu_{A}([0, T]) \inf _{j \geq 1} \int_{0}^{t}\left\|f_{\alpha}^{i}(s, \omega)-g_{\alpha}^{j}(s, \omega)\right\|^{2} \mathrm{~d} A_{s}(\omega) \text { a.s. } \\
& =\mu_{A}([0, T]) \int_{0}^{t} \inf _{j \geq 1}\left\|f_{\alpha}^{i}(s, \omega)-g_{\alpha}^{j}(s, \omega)\right\|^{2} \mathrm{~d} A_{s}(\omega) \text { a.s. }
\end{aligned}
$$

Then 


$$
\begin{aligned}
& \mu_{A}([0, T]) \int_{0}^{t} \inf _{j \geq 1}\left\|f_{\alpha}^{i}(s, \omega)-g_{\alpha}^{j}(s, \omega)\right\|^{2} \mathrm{~d} A_{s}(\omega) \\
& \leq \mu_{A}([0, T]) \int_{0}^{t} \sup _{i \geq 1} \inf _{j \geq 1}\left\|f_{\alpha}^{i}(s, \omega)-g_{\alpha}^{j}(s, \omega)\right\|^{2} \mathrm{~d} A_{s}(\omega) \\
& \leq \mu_{A}([0, T]) \int_{0}^{t} d_{H}^{2}\left([F(s, \omega)]^{\alpha},[G(s, \omega)]^{\alpha}\right) \mathrm{d} A_{s}(\omega) \\
& \leq \mu_{A}([0, T]) \int_{0}^{t} d_{\infty}^{2}(F(s, \omega), G(s, \omega)) \mathrm{d} A_{s}(\omega) \text { a.s. }
\end{aligned}
$$

Then

$$
\operatorname{supinf}_{i \geq 1}\left\|\int_{j \geq 1}^{t} f_{\alpha}^{i}(s, \omega) \mathrm{d} A_{s}(\omega)-\int_{0}^{t} g_{\alpha}^{j}(s, \omega) \mathrm{d} A_{s}(\omega)\right\|^{2} \leq \mu_{A}([0, T]) \int_{0}^{t} d_{\infty}^{2}(F(s, \omega), G(s, \omega)) \mathrm{d} A_{s}(\omega) \text { a.s. }
$$

Similarly, we have

$$
\operatorname{supinf}_{j \geq 1}\left\|\int_{i}^{t} g_{\alpha}^{j}(s, \omega) \mathrm{d} A_{s}(\omega)-\int_{0}^{t} f_{\alpha}^{i}(s, \omega) \mathrm{d} A_{s}(\omega)\right\|^{2} \leq \mu_{A}([0, T]) \int_{0}^{t} d_{\infty}^{2}(F(s, \omega), G(s, \omega)) \mathrm{d} A_{s}(\omega) \text { a.s. }
$$

Then for each $\alpha \in[0,1]$

$$
d_{H}^{2}\left(\int_{0}^{t}[F(s, \omega)]^{\alpha} \mathrm{d} A_{s}(\omega), \int_{0}^{t}[G(s, \omega)]^{\alpha} \mathrm{d} A_{s}(\omega)\right) \leq \mu_{A}([0, T]) \int_{0}^{t} d_{\infty}^{2}(F(s, \omega), G(s, \omega)) \mathrm{d} A_{s}(\omega) \text { a.s. }
$$

Moreover

$$
\sup _{\alpha \in[0,1]} d_{H}^{2}\left(\int_{0}^{t}[F(s, \omega)]^{\alpha} \mathrm{d} A_{s}(\omega), \int_{0}^{t}[G(s, \omega)]^{\alpha} \mathrm{d} A_{s}(\omega)\right) \leq \mu_{A}([0, T]) \int_{0}^{t} d_{\infty}^{2}(F(s, \omega), G(s, \omega)) \mathrm{d} A_{s}(\omega) a . s .
$$

Hence

$$
d_{\infty}^{2}\left(\int_{0}^{t} F(s, \omega) \mathrm{d} A_{s}(\omega), \int_{0}^{t} G(s, \omega) \mathrm{d} A_{s}(\omega)\right) \leq \mu_{A}([0, T]) \int_{0}^{t} d_{\infty}^{2}(F(s, \omega), G(s, \omega)) \mathrm{d} A_{s}(\omega) \text { a.s. }
$$

Remark 1. In Theorem 6 and Theorem 7, the inequalities hold too if we take the expectation on both sides.

\section{Conclusion}

In [21], the author studied the Lebesgue-Stieltjes integral of real stochastic processes with respect to fuzzy valued stochastic processes. In some references such as [5] [6], the integrals of fuzzy stochastic processes with respect to time $t$ and Brownian motion were studied. In order to guarantee measurability of the integral, Kim (2005) Li and Ren (2007) defined the integral indirectly by taking the decomposable closure. Here, when the integrand taked value in compact and convex subsets of $F\left(R^{d}\right)$, we defined directly the integral of fuzzy stochastic process with respect to real-valued finite variation processes by using selection method, which is different from the above references. Then we proved the measurability (Theorem 2), which was key and guaranteed the reasonability of the definition. Attribute to the good property of finite variation of integrator, the integral was bounded as and $L^{2}$-bounded under the metric $d_{\infty}$ (Theorem 3, Theorem 6 and Theorem 7). This property was much well than the integral with respect to Brownian motion since the latter was of infinite variation. Thanks to the boundedness of the integral, it was possible to do the further work such as exploring solutions of fuzzy stochastic differential equations.

\section{Acknowledgements}

We thank the editor and the referees for their comments. This work is partly supported by NSFC (No. 11371135).

\section{References}

[1] Puri, M.L. and Ralescu, D.A. (1986) Fuzzy Random Variables. Journal of Mathematical Analysis and Applications, 114, 409-422. http://dx.doi.org/10.1016/0022-247X(86)90093-4

[2] Kisielewicz, M. (1997) Set-Valued Stochastic Integrals and Stochastic Inclusions. Stochastic Analysis and Applications, 15, 780-800. http://dx.doi.org/10.1080/07362999708809507 
[3] Kim, B.K. and Kim, J.H. (1999) Stochastic Integrals of Set-Valued Processes and Fuzzy Processes. Journal of Mathematical Analysis and Applications, 236, 480-502. http://dx.doi.org/10.1006/jmaa.1999.6461

[4] Jung, E.J. and Kim, J.H. (2003) On Set-Valued Stochastic Integrals. Stochastic Analysis and Applications, 21, $401-418$. http://dx.doi.org/10.1081/SAP-120019292

[5] Kim, J.H. (2005) On Fuzzy Stochastic Differentials Equations. Journal of the Korean Mathematical Society, 42, 153169. http://dx.doi.org/10.4134/JKMS.2005.42.1.153

[6] Li, S. and Ren, A. (2007) Representation Theorems, Set-Valued and Fuzzy Set-Valued ITO Integral. Fuzzy Sets and Systems, 158, 949-962. http://dx.doi.org/10.1016/j.fss.2006.12.004

[7] Michta, M. (2011) On Set-Valued Stochastic Integrals and Fuzzy Stochastic Equations. Fuzzy Sets Systems, 177, 1-19. http://dx.doi.org/10.1016/j.fss.2011.01.007

[8] Zhang, J., Li, S., Mitoma, I. and Okazaki, Y. (2009) On Set-Valued Stochastic Integrals in an M-Type 2 Banach Space. Journal of Mathematical Analysis and Applications, 350, 216-233. http://dx.doi.org/10.1016/j.jmaa.2008.09.017

[9] Zhang, J., Li, S., Mitoma, I. and Okazaki, Y. (2009) On the Solution of Set-Valued Stochastic Differential Equations in M-Type 2 Banach Space. Tohoku Mathematical Journal, 61, 417-440. http://dx.doi.org/10.2748/tmj/1255700202

[10] Li, J. and Wang, J. (2012) Fuzzy Set-Valued Stochastic Lebesgue Integral. Fuzzy Sets and Systems, 200, 48-64. http://dx.doi.org/10.1016/j.fss.2012.01.021

[11] Mitoma, I., Okazaki, Y. and Zhang, J. (2010) Set-Valued Stochastic Differential Equations in M-Type 2 Banach Space. Communications on Stochastic Analysis, 4, 215-237.

[12] Zhang, J., Mitoma, I. and Okazaki, Y. (2013) Set-Valued Stochastic Integral with Respect to Poisson Process in a Banach Space. International Journal of Approximate Reasoning, 54, 404-417.

[13] Fei, W. (2013) Existence and Uniquess for Solutions to Fuzzy Stochastic Differential Equations Driven by Local Martingales under the Non-Lipschtiz Condition. Nolinear Analysis, 76, 202-214. http://dx.doi.org/10.1016/j.na.2012.08.015

[14] Zhang, J. and Qi, J. (2013) Set-Valued Stochastic Integrals with Respect to Finite Variation Processes. Advances in Pure Mathematics, 3, 15-19. http://dx.doi.org/10.4236/apm.2013.39A1003

[15] Hu, S. and Papageorgiou, N. (1997) Handbook of Multivalued Analysis, Volume I: Theory. Kluwer Academic Publishers, Boston.

[16] Negoito, C. and Ralescu, D. (1975) Applications of Fuzzy Sets to Systems Analysis. Wiley, New York. http://dx.doi.org/10.1007/978-3-0348-5921-9

[17] Kwakernaak, H. (1987) Fuzzy Random Variables, Definition and Theoroems. Information Sciences, 15, 1-29. http://dx.doi.org/10.1016/0020-0255(78)90019-1

[18] Zhang, W., Li, S. and Wang, Z. (2007) Set-Valued Stochastic Process Introduction. Science Press, Beijing. (In Chinese)

[19] Charalambos, D.A. and Kim, C.B. (1994) Infinite Dimensional Analysis. Springer-Verlag, Berlin.

[20] Li, S., Ogura, Y. and Kreinovich, Y. (2002) Limit Theorems and Applications of Set-valued and Fuzzy Set-Valued Random Variables. 43rd Edition, Kluwer Academic Publishers, Dordrecht. http://dx.doi.org/10.1007/978-94-015-9932-0

[21] Feng, Y. (2001) Fuzzy-Valued Mappings with Finite Variation, Fuzzy-Valued Measures and Fuzzy-Valued Lebesgue-Stieltjes Integrals. Fuzzy Sets and Systems, 2, 227-236. http://dx.doi.org/10.1016/S0165-0114(99)00178-5 